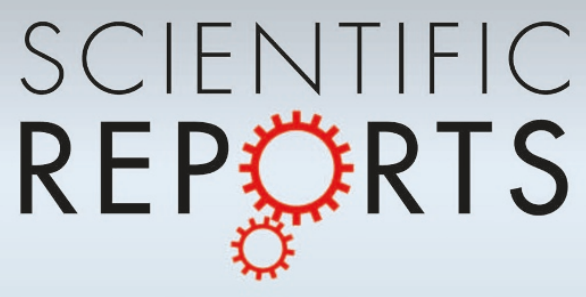

OPEN

SUBJECT AREAS:

DESIGN, SYNTHESIS AND

PROCESSING

OPTICAL MANIPULATION AND TWEEZERS

OPTICAL PHYSICS

NONLINEAR OPTICS

Received

14 June 2013

Accepted

27 March 2014

Published

2 May 2014

Correspondence and requests for materials should be addressed to L.C. (chenlx@xmu.edu.

cn)

\title{
Polarization singularities and orbital angular momentum sidebands from rotational symmetry broken by the Pockels effect
}

\author{
Xiancong $\mathrm{Lu}^{1,2}$, Ziwen $\mathrm{Wu}^{1}$, Wuhong Zhang' \& Lixiang Chen'
}

\begin{abstract}
'Department of Physics and Laboratory of Nanoscale Condensed Matter Physics, Xiamen University, Xiamen 361005, China, ${ }^{2}$ Institute of Theoretical Physics and Astrophysics, Xiamen University, Xiamen 361005, China.
\end{abstract}

\begin{abstract}
The law of angular momentum conservation is naturally linked to the rotational symmetry of the involved system. Here we demonstrate theoretically how to break the rotational symmetry of a uniaxial crystal via the electro-optic Pockels effect. By numerical method based on asymptotic expansion, we discover the 3D structure of polarization singularities in terms of $C$ lines and $L$ surfaces embedded in the emerging light. We visualize the controllable dynamics evolution of polarization singularities when undergoing the Pockels effect, which behaves just like the binary fission of a prokaryotic cell, i.e., the splitting of $C$ points and fission of $L$ lines are animated in analogy with the cleavage of nucleus and division of cytoplasm. We reveal the connection of polarization singularity dynamics with the accompanying generation of orbital angular momentum sidebands. It is unexpected that although the total angular momentum of light is not conserved, the total topological index of $C$ points is conserved.
\end{abstract}

T he study of polarization of light has a long history. Nowadays polarization has been of broad applications in many areas of science and technology, ranging from physics to biology and chemistry ${ }^{1}$. Recent interest may be traced back to the seminal work by Nye who revealed the generic structure of polarization singularities ${ }^{2-4}$. In the general 3D picture, there are two types of polarization singularities: lines along which the polarization is purely circular (Clines) and surfaces on which the polarization is purely linear ( $L$ surfaces), where the orientation and handedness of polarization ellipse are indefinite, respectively. In the context of singular optics, polarization singularities are regularly considered as the vector analog of phase singularities or optical vortices in scalar fields $s^{5}$. Circular polarizations are in essence associated with spin angular momentum of photons, while optical vortices are often studied with twisted photons carrying quantized orbital angular momentum $(\mathrm{OAM})^{6,7}$. It has been demonstrated that manipulating optical beams with vortex lines in the forms of knots or links holds promise for future laser technology and optical trapping schemes ${ }^{8-10}$. Beyond the uniform polarization in scalar fields, the morphology and topology of polarization singularities in vector fields are much richer and subtler, as predicted by Dennis and later verified by Flossmann et $a^{11,12}$. Recent years have witnessed a rapidly growing interest in these amazing structures, which are found to appear in the skylight ${ }^{13}$, isotropic microchip laser ${ }^{14}$, near field nanooptics $^{15}$, and inhomogeneous anisotropic plates ${ }^{16}$.

Here we report theoretically a rather fascinating phenomenon that in a uniaxial crystal when undergoing Pockels effect, the tunable evolution of polarization singularities of emerging light behaves just like the binary fission, such as in a prokaryotic cell division ${ }^{17}$. Under the control of an externally applied electric field, the splitting of $C$ points and fission of $L$ lines can be depicted vividly in analogy with the cleavage of nucleus and division of cytoplasm. Polarization singularities in crystals have been indeed studied extensively ${ }^{18-21}$, but apparently seldom considering the Pockels effect ${ }^{22,23}$. Previously, we demonstrated the capability of using Pockels effect to manipulate spin and orbital angular momentum in optically active crystals or electro-optic birefrigent $\mathrm{crystals}^{24-27}$. In contrast, we here aim to show another phenomenon of tunable polarization singularities by electro-optically breaking the rotational symmetry of a uniaxial crystal, which therefore lends itself to a flexible and realtime manipulation. Our work can also be connected to those reporting the conservation law of angular momentum related to the rotational symmetry, such as in isotropic crystals or uniaxial crystals $\mathrm{s}^{26,28-31}$. In contrast, here the rotational symmetry around the optic axis is slightly broken by the applied electric field, since the specific 
(a)

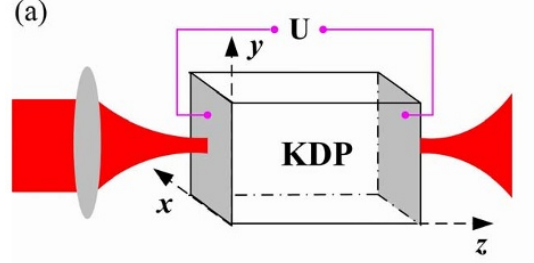

(b)

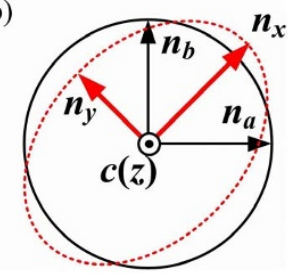

Figure $1 \mid$ The theoretical scheme. (a) The proposed schematic diagram. (b) The deformed ellipse of refractive index of KDP when undergoing the Pockels effect.

second-order susceptibilities $\chi_{x y z}$ and $\chi_{y x z}$ are activated to respond for the deformation of the refractive index ellipsoid of electro-optic crystal. Furthermore, we reveal the connection of our observations with the accompanying generation of OAM sidebands. OAM sidebands have been found intrinsic to reflection due to Goos-Hanchen and Imbert-Fedorov shifts ${ }^{32}$. But ours is resulted from transverse angular anisotropy induced by Pockels effect. Surprisingly, total angular momentum of light is not conserved, whereas total topological index of $C$ points is conserved.

Our scheme is sketched in Figure 1. We consider a $z$-cut uniaxial crystal of potassium dihydrogen phosphate (KDP), which is a typical electro-optic material belonging to class $\overline{4} 2 \mathrm{~m}$. The principle refractive indices $n_{o}=1.5074, n_{e}=1.4669$, and the nonvanishing electrooptic coefficients $\gamma_{41}=\gamma_{52}=8, \gamma_{63}=11$ (in pm/V), respectively $y^{33}$. Assume the initial light beam of wavelength $\lambda=1.633 \mu \mathrm{m}$ is a lefthanded circularly polarized one propagating along the optic axis, namely, $\mathbf{E}\left(\mathbf{r}_{\perp}, z=0\right)=\exp \left(-r_{\perp}^{2} / 2 s^{2}\right) \hat{\mathbf{e}}_{+}$, where $s=4.59 \mu \mathrm{m}$ is the beam waist and $\hat{\mathbf{e}}_{+}=\frac{1}{\sqrt{2}}[1, \mathrm{i}]$ as well as $\hat{\mathbf{e}}_{-}=\frac{1}{\sqrt{2}}[1,-i]$ forms the circular bases. The incident and exit interfaces of KDP are both coated with transparent electrode in order to apply a longitudinal electric field $\mathbf{E}_{0}=E_{0} \hat{e}_{z}$. When $E_{0}$ is switched on, a nonlinear polarization responsible for the Pockels effect is induced ${ }^{34}: \mathbf{P}^{E O}=$ $2 \varepsilon_{0} \chi^{(2)}(\omega, 0)$ : $\mathrm{EE}_{0}$, where $\varepsilon_{0}$ is the permittivity of free space, $\chi^{(2)}(\omega$, $0)$ the second-order susceptibility tensor related to the Pockels effect, and $\mathrm{E}$ the light field of frequency $\omega$. Starting from Maxwell equations and considering total electric displacement $\mathbf{D}=\varepsilon_{0} \vec{\varepsilon}_{r} \mathbf{E}+\mathbf{P}^{E O}$, we have the following equation governing the complex amplitude of a propagating light,

$$
\nabla^{2} \mathbf{E}-\nabla(\nabla \cdot \mathbf{E})+k_{0}^{2} \overleftrightarrow{\varepsilon}_{r} \mathbf{E}+\mu_{0} \omega^{2} \mathbf{P}^{E O}=0,
$$

where $k_{0}=2 \pi / \lambda, \overleftrightarrow{\varepsilon}_{r}=\operatorname{diag}\left(n_{o}^{2}, n_{o}^{2}, n_{e}^{2}\right)$ denotes the relative dielectric tensor, and $\mu_{0}$ is the magnetic susceptibility in vacuum.

In this paper, we follow Ciattoni's angular spectrum representation method ${ }^{35}$ and our recently developed numerical method based on asymptotic expansion ${ }^{36}$ to calculate the complex vectorial field $\mathrm{E}\left(\mathbf{r}_{\perp}, z\right)$ in the propagating space. As $\mathrm{E}\left(\mathbf{r}_{\perp}, z\right)$ is obviously position dependent, it is naturally expected that the emerging polarization from KDP is also spatially variant. Besides, the polarization should be electrically tunable by $E_{0}$ via the Pockels effect. In general, the geometry of polarization ellipse can be completely described in terms of four Stokes parameters ${ }^{37}: S_{0}=E_{x} E_{x}^{*}+E_{y} E_{y}^{*}, S_{1}=E_{x} E_{x}^{*}-E_{y} E_{y}^{*}$, $S_{2}=2 \operatorname{Re}\left(E_{x}^{*} E_{y}\right)$, and $S_{3}=2 \operatorname{Im}\left(E_{x}^{*} E_{y}\right)$. Physically, the orientation of major axis and the ellipticity of polarization ellipse are characterized by $\theta=\frac{1}{2} \arg \left(S_{1}+i S_{2}\right)$ and $\varepsilon=S_{3} / S_{0}$, respectively. In very cross section, $L$ lines are those on which $S_{3}=0, C$ points are defined as the intercept of the loci $S_{1}=0$ and $S_{2}=0$. Besides, $C$ points can be classified into some basic types, such as lemon, monstar and $\operatorname{star}^{5,11}$.

\section{Results}

We plot in Figure 2 our numerical solution of the 3D polarization singularities embedded in the emerging light. It is found that when

the KDP crystal is undergoing the Pockels effect, $C$ lines and $L$ surfaces attain different morphologies. Figure 2(a) demonstrate the simple case when $E_{0}$ is absent. It looks like a right circular cone with the conical surface being $L$ surface and the axis of the cone being $C$ line. As the light propagates, the $C$ line is stretching along the propagation direction coinciding with optical axis. While $E_{0}$ is switched on, however, the $\mathrm{Cline}$ is quickly bifurcated into two ones, appearing like a pair of compasses with both arms being left-handed circular polarization and deflecting from the optic axis. Besides, the $L$ surface is then gradually cleaved into two separate sleeves and each encircles one $\mathrm{C}$ line. By a comparison of Figure 2(a)-2(d), we find that applying a larger $E_{0}$ accelerates the cleavage of $L$ surface. As $E_{0}$ increases from $5.31 \mathrm{kV} / \mathrm{cm}$ to $10.62 \mathrm{kV} / \mathrm{cm}$, the cleavage point is brought forward from $z=6000 \mu m$ to $3000 \mu m$ or so.

Figure 2 shows only the frame of the 3D polarization structures. One can image that the volume is filled with many polarization ellipses of various shapes and orientations. For a better view, we also visualize their 2D fine structures in Figure 3, assuming the length of KDP crystal is fixed at $z=6000 \mu \mathrm{m}$. By tuning $E_{0}$, we observe a fascinating phenomenon that the dynamic evolution of polarization singularities when undergoing the Pockels effect just behaves like the binary fission of a prokaryote cell ${ }^{17}$. In Figure 3(a), the KDP crystal is pure uniaxial without disturbance $\left(E_{0}=0\right)$. So the central $C$-point is simply surrounded by one $L$ circle. Here we use " $L$ cell" to describe the region that the $L$ line embraces. As $E_{0}$ is increasing, the splitting of $C$ points and fission of $L$ lines can be animated in analogy with the cleavage of nucleus and division of membrane in a cell division. Specifically, the $C$ point first replicates (like a single DNA), then attaches each copy to a different part of $L$ cell. In Figure 3(b) with $E_{0}=5.31 \mathrm{kV} / \mathrm{cm}$, the $L$ cell begins to elongate along $x$ direction, and the original and replicate $C$ points are pulled apart to separate poles. Then the middle portion of the $L$ cell begins to sink, and a cross wall is well developed and formed at $E_{0}=6.90 \mathrm{kV} / \mathrm{cm}$ in Figure 3(c). When $E_{0}=10.62 \mathrm{kV} / \mathrm{cm}$, the $L$ cell has been completely spit into two daughters of identical C point, shown in Figure 3(d). Obviously, present manipulation on polarization singularities could be flexible and fast, since the electro-optic Pockels effect possesses a responsible time less than one nanosecond ${ }^{38}$.

It is crucial for us to reveal the underlying reasons that support the above interesting features. By analogy between polarization and phase singularities, we attribute this to the accompanying angular momentum dynamics. In principle, we can express the transverse light field in terms of both circular polarizations and spiral harmonics,

$$
\mathbf{E}_{\perp}\left(\mathbf{r}_{\perp}, z\right)=\sum_{l=-\infty}^{+\infty}\left[E_{l}^{+}\left(r_{\perp}, z\right) \hat{\mathbf{e}}_{+}+E_{l}^{-}\left(r_{\perp}, z\right) \hat{\mathbf{e}}_{-}\right] \exp (i l \phi),
$$

and $W_{l}^{ \pm}=\frac{1}{\eta} \int_{0}^{\infty} 2 \pi r\left|E_{l}^{ \pm}\left(r_{\perp}, z\right)\right|^{2} d r$ (with $\eta$ being the normalized constant) can thus be interpreted as the weight of each OAM mode. As an echo of Figure 3, we show the numerical results of $W_{l}^{ \pm}$in Figure 4. In Figure 4(a), the initial left-handed circularly polarized light with $l$ $=0$ is partially converted into right-handed one with $l=2$ while acquires $2 \hbar \mathrm{OAM}$ per photon, and therefore conserving total angular momentum. This is just the case for a pure uniaxial crystal $^{29,30}$. When undergoing Pockels effect, besides the energy transfer from left- to right-handed circular component, we find that the energy for each circular component is distributed over several neighboring even OAM modes, i.e., the OAM sidebands are generated due to mode coupling. For left-handed component, the main coupling is to the $l=$ \pm 2 modes, with a small efficiency of $1.7 \%, 2.7 \%$ and $4.7 \%$ in Figure 4(b), 4(c) and 4(d), respectively; while those to other higher modes are even weaker, as interfered from insets of Figure 4. For right-handed component, the significant mode coupling occurs 
(a)

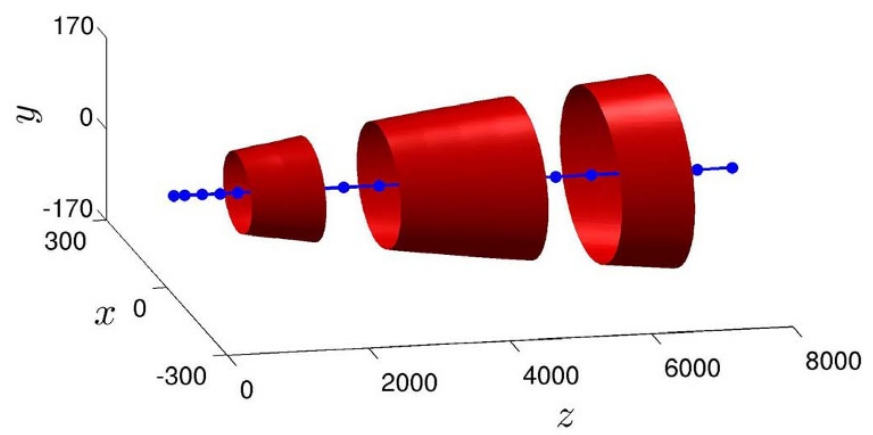

(c)

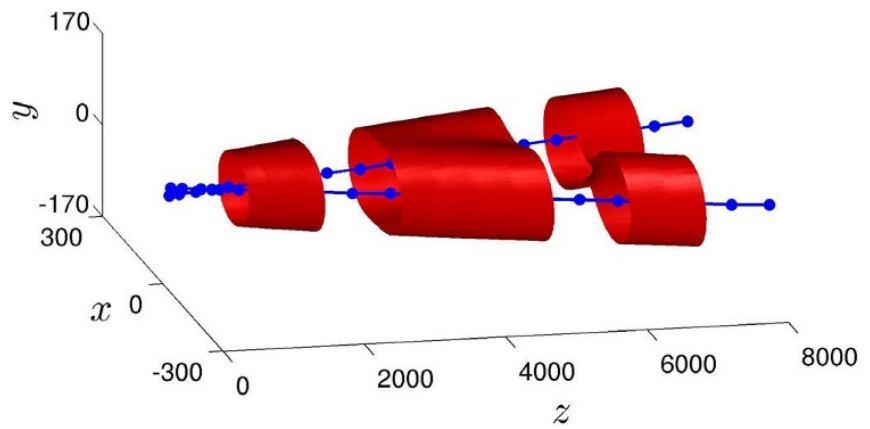

$(b)$

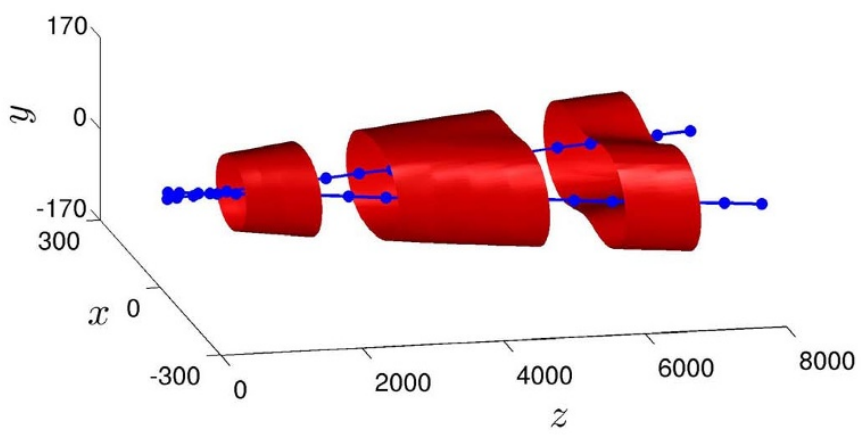

$(d)$

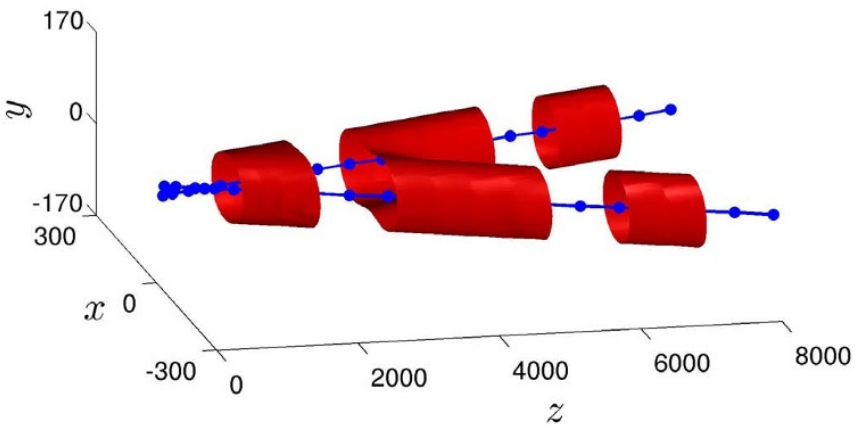

Figure $2 \mid$ The 3D structures of $C$ lines (blue) and $L$ surfaces (red). Different electric fields are applied: (a) $E_{0}=0,(\mathrm{~b}) E_{0}=5.31 \mathrm{kV} / \mathrm{cm},(\mathrm{c}) E_{0}=6.90 \mathrm{kV} /$ $\mathrm{cm}$ and (d) $E_{0}=10.62 \mathrm{kV} / \mathrm{cm}$. All coordinates are in unit of $\mu \mathrm{m}$. See also the Supplemental information video 1 .
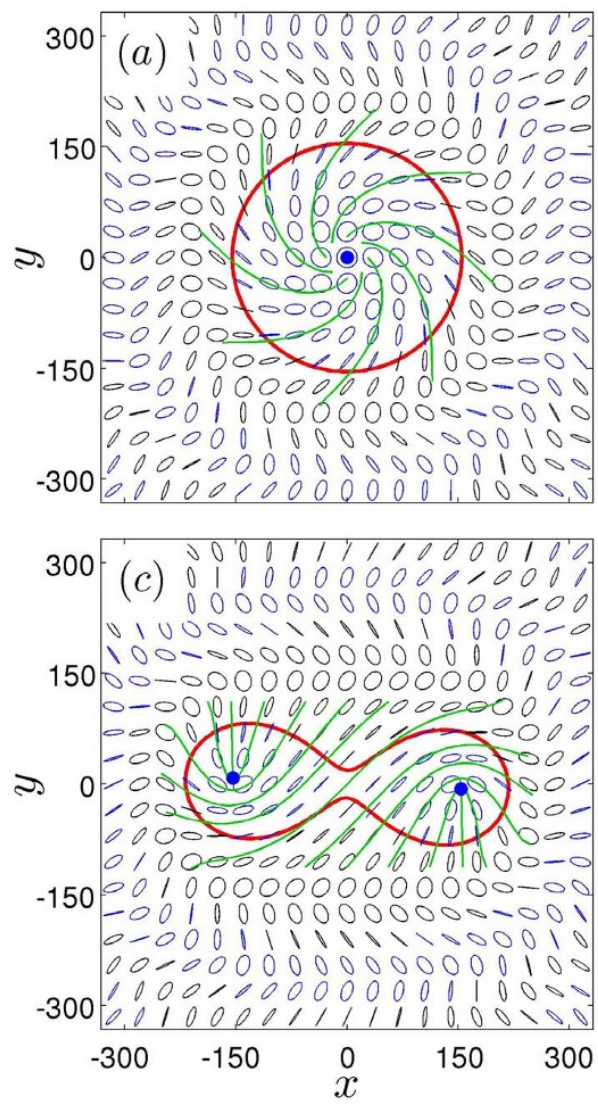

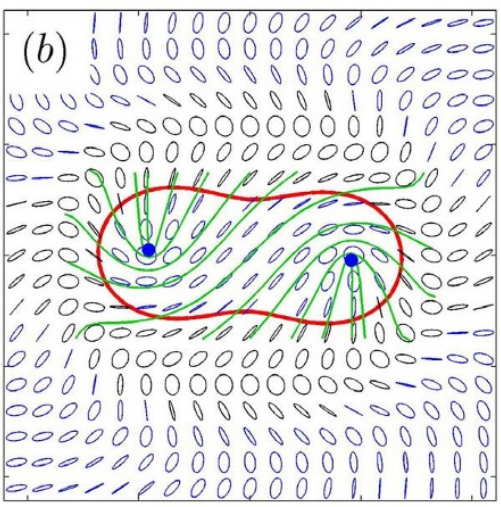

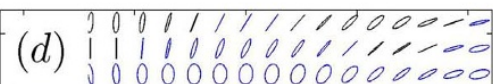
u u u 0 o o a d o d 00000 $0000-7 D N 1111111000$ $00-0000000000000110$ - 000100000000000001 $000 \mathrm{H}+10 \% 0 / 000000000$ - Y 12000 00 (e) 10000 of 1000

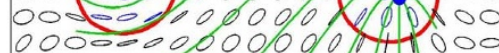
l 10000000000 dolobob $110000000000000-00$

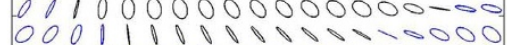
0000000000000000000 0000000000000000000 $00-1,1,10000000011111110$ $0-000011,1,110.00011 .1$ $\begin{array}{lllll}-300 & -150 & 0 & 150 & 300\end{array}$

Figure $3 \mid$ The 2D fine structure of $C$ points (blue dots) and $L$ lines (red lines). Green lines denote the streamlines of major axis of polarization ellipse. Under the control of $E_{0}$, they behave like the binary fission of a prokaryotic cell, where $E_{0}$ is the same as those in Figure 2. See also the supplementary information video 2 . 

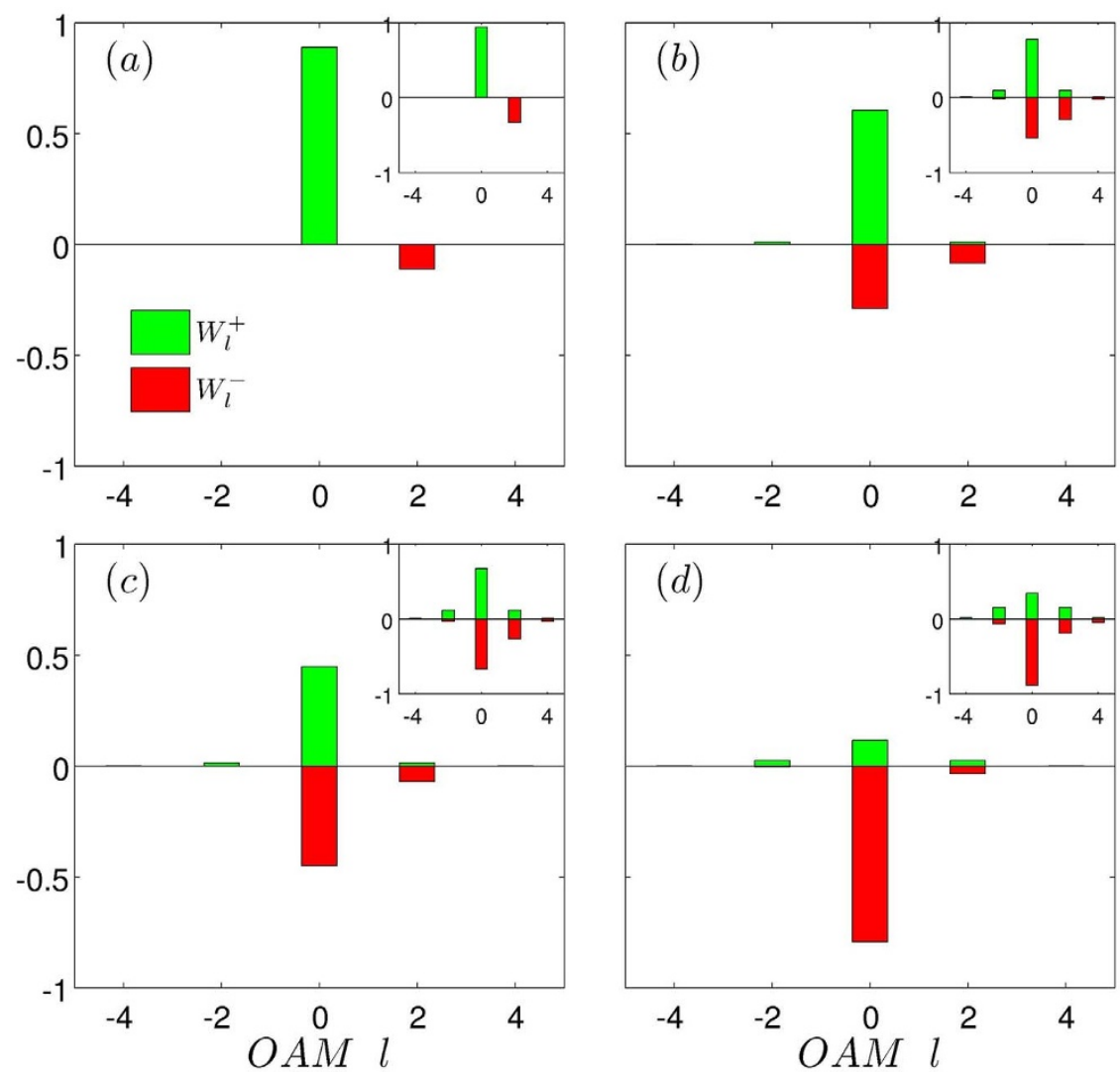

Figure $4 \mid$ The accompanying generation of OAM sidebands. Green and red bars denote the sidebands for left- and right-handed components, respectively, where $E_{0}$ is the same as those in Figures 2 and 3. Insets are the pseudo sidebands of $\sqrt{W_{l}^{ \pm}}$, which "exaggerate" the weak coupling to higher even OAM of $l= \pm 4$.

between $l=0$ and $l=+2$. Besides, it is electrically tunable. As has been revealed by Angelsky et $a l^{39}$, there is a relationship between topological characteristics of component vortices and polarization singularities, namely, $C$ points locate at the vortices of the opposite circular component. In our case, the left-handed $C$ points are coming from the vortices of right-handed component. For a pure uniaxial crystal, only $l=+2$ vortex exists such that only a left-handed $C$ points emerges. It is just the superposition of $l=0$ and $l=+2$ modes that accounts for the formation of a pair of right-handed component vortices, and therefore, the formation of a pair of left-handed $C$ points. As $E_{0}$ is increasing, the intensity ratio of $l=+2$ to $l=0$ decreases from $30 \%, 16 \%$ to $5 \%$, shown in Figure 4(b) to 4(d). As a consequence, two vortices are pushed away, so are the $C$ points, see Figure $3(\mathrm{~b})$ to $3(\mathrm{~d})$.

\section{Discussion}

The above generation of OAM sidebands can be well understood from the transverse angular anisotropy induced by the Pockels effect. According to the refractive index ellipsoid theory ${ }^{33}$, we know that, with the application of electric field along $z$ direction, the transverse isotropy of $n_{x}=n_{y}=n_{o}$ cannot hold anymore; instead, $n_{x}=n_{o}-$ $n_{o}^{3} \gamma_{63} E_{0} / 2$ and $n_{y}=n_{o}+n_{o}^{3} \gamma_{63} E_{0} / 2$. Thus we can define the quantity, $\Delta=n_{x}-n_{y}=n_{o}^{3} \gamma_{63} E_{0}$, to characterize the broken degree of rotational invariance around the optic axis, which is evidently proportional to $E_{0}$. As is well known, the conservation law of angular momentum is naturally linked with the rotational symmetry. So here we expect that applying a larger $E_{0}$ will give rise to a larger nonconservative amount of angular momentum. Generally, the angular momentum per photon within emerging light can be expressed as a sum of spin and orbital parts, namely, $J=\sum_{l}(l+1) W_{l}^{+}+\sum_{l}(l-1) W_{l}^{-}$. By calculation, we obtain the angular momentum change (after subtracting $\hbar$ for initial left-handed light): $\Delta J=0 \hbar, \Delta J=$ $-0.5794 \hbar, \Delta J=-0.9015 \hbar$ and $\Delta J=-1.6008 \hbar$ for Figure 4(a)$4(\mathrm{~d})$, respectively, thereby confirming our prediction.

But, surprisingly enough, the total topological index of $C$ points is preserved. In Figure 3(a), the polarization streamlines make up spiral branches. As we make a complete circuit clockwise around $C$ point, note that the polarization ellipse rotates clockwise through a complete revolution. Consequently, the topological index is $I_{\mathrm{C}}=+1$, and this corresponds to the double degeneracy of the central $C$ point. While in Figure 3(b)-3(d), the signed number of turns that the streamlines makes around each $C$ point is $+1 / 2$, and the number of streamlines that terminate on the $C$ point is 1 , so each $C$ point is a lemon type ${ }^{11}$. Therefore, the total topological index is preserved, namely, $I_{\mathrm{C}}=1 / 2+1 / 2=1$, despite that $E_{0}$ is changing. A complex Stokes field, $\sigma=S_{1}+i S_{2}=|\sigma| \exp (i 2 \theta)$ ( $\theta$ is the orientation of polarization ellipse), is usefully defined to study the Stokes vortex ${ }^{40}$. It follows that the index of $C$ points $I_{C}$ is just half the charge of the Stokes vortex. As can be seen from Figure 4, only and always the right-handed vortex of charge 2 dominates in the OAM sidebands such that $I_{\mathrm{C}}=1$ remain preserved in each subfigure.

In conclusion, we have discovered and visualized the interesting dynamics evolution of polarization singularities for a light field emerging from a uniaxial crystal undergoing the Pockels effect, where the splitting of $C$ points and fission of $L$ lines are animated in analogy with the cleavage of nucleus and division of cytoplasm in the binary fission. Because of the rotational symmetry breaking, we find that the total angular momentum of light is not conserved, but unexpectedly, the total topological index of $\mathrm{C}$ points is conserved. We reveled the connection of these findings with the accompanying generation of OAM sidebands, as a result of the OAM mode coupling induced by the Pockels effect. Our results may supply another perspective of angular momentum conservation law in the context of 
(a)

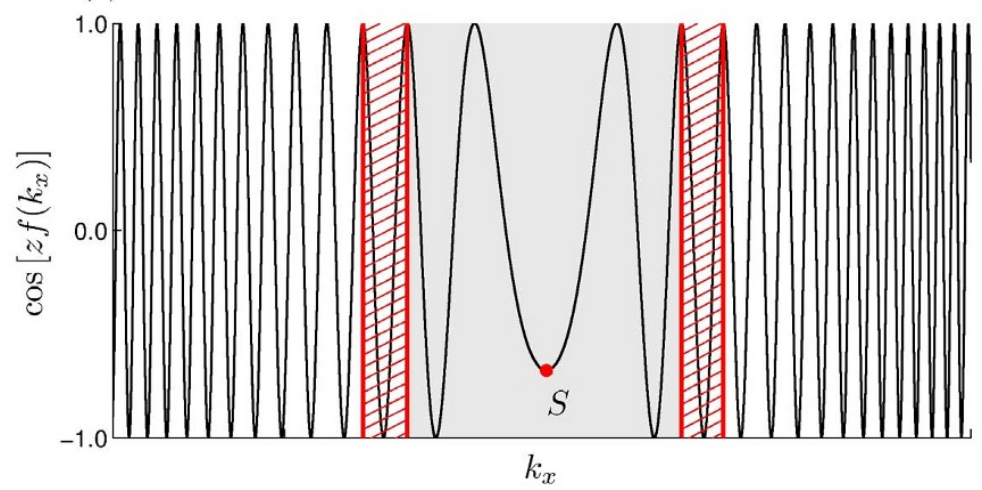

(b)

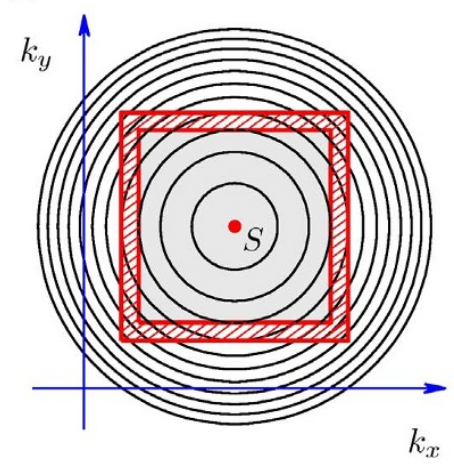

Figure $5 \mid$ The schematic diagram for the domain of integration. (a) The oscillatory integrand $\cos \left[z f\left(k_{x}\right)\right]$ is plotted in the $k_{x}$ direction. (b) Top view of integrating boundary in two-dimensional Fourier space, where the concentric circles are the oscillating periods of the integrand. In both (a) and (b), the red point $S$ denotes the stationary point of function $f\left(k_{x}, k_{y}\right)$, the gray area centered at point $S$ denotes the domain of integration, and the shading region with red slant lines denotes one oscillating period where a coarse-grained average is performed.

rotational symmetry breaking, and provide a flexible and fast manipulation on the polarization singularities.

\section{Methods}

According to the refractive index ellipsoid theory ${ }^{33}$, with the application of longitudinal electric field $E_{0} \hat{e}_{z}$, the refractive index ellipsoid of KDP is deformed as, $a^{2} / n_{o}^{2}+b^{2} / n_{o}^{2}+c^{2} / n_{e}^{2}+2 \gamma_{63} E_{0} a b=1$, where $a, b$, and $c$ denote the crystalline axes. Considering the symmetry of $a$ and $b$ in the ellipsoid equation, we choose a new coordinate system $x, y$, and $z$, where $x$ and $y$ are related to $a$ and $b$ by $45^{\circ}$ rotation while $z$ is parral to $c$, as illustrated in Fig. 1(b). Then the equation of index ellipsoid becomes $^{33}, x^{2}\left(1 / n_{o}^{2}+\gamma_{63} E_{0}\right)+y^{2}\left(1 / n_{o}^{2}-\gamma_{63} E_{0}\right)+z^{2} / n_{e}^{2}=1$. By considering the nonlinearity induced by the Pockels effect as a perturbation, namely, $\gamma_{63} E_{0}<<n_{0}^{-2}$, we know that the principle indices of the new index ellipsoid are approximately: $n_{x}=n_{o}-n_{o}^{3} \gamma_{63} E_{0} / 2, n_{y}=n_{o}+n_{o}^{3} \gamma_{63} E_{0} / 2$ and $n_{z}=n_{e}$, where the electro-optically induced biaxial anisotropy of $n_{x} \neq n_{y} \neq n_{z}$ just accounts for the broken rotational symmetry, as discussed above. Besides, in light of such an approximation, we are able to present an efficient numerical solution to Eq. (1) based on the Ciattoni's theory of angular spectrum representation and the method of asymptotic expansion.

The key idea of Ciattoni's angular spectrum representation method is to express the light field $\mathbf{E}(\mathbf{r})=\mathbf{E}\left(\mathbf{r}_{\perp}, z\right)$ using two-dimensional Fourier transformation ${ }^{35}$,

$$
\mathbf{E}\left(\mathbf{r}_{\perp}, z\right)=\int d^{2} \mathbf{k}_{\perp} \exp \left(i \mathbf{k}_{\perp} \cdot \mathbf{r}_{\perp}\right) \tilde{\mathbf{E}}\left(\mathbf{k}_{\perp}, z\right),
$$

where $\mathbf{r}_{\perp}=x \hat{\mathbf{e}}_{x}+y \hat{\mathbf{e}}_{y}$ and $\mathbf{k}_{\perp}=k_{x} \hat{\mathbf{e}}_{x}+k_{y} \hat{\mathbf{e}}_{y}$ are vectors in the real and momentum space, respectively. Along this line, to solve Eq. (1), we expand the light field $\mathrm{E}\left(\mathbf{r}_{\perp}, z\right)$ in the coordinate system $a, b, c$ by 2-D Fourier transformation, and similarly the nonlinear polarization $\mathbf{P}^{E O}\left(\mathbf{r}_{\perp}, z\right)=\int d^{2} \mathbf{k}_{\perp} \exp \left(i \mathbf{k}_{\perp} \cdot \mathbf{r}_{\perp}\right) \tilde{\mathbf{P}}^{E O}\left(\mathbf{k}_{\perp}, z\right)$, whose components in momentum space are: $\tilde{P}_{a}^{E O}=-\varepsilon_{0} n_{o}^{4} \gamma_{63} E_{0} \tilde{E}_{b}, \tilde{P}_{b}^{E O}=-\varepsilon_{0} n_{o}^{4} \gamma_{63} E_{0} \tilde{E}_{a}$, and $\tilde{P}_{c}^{E O}=0$. Substituting these transformations into Eq. (1), using the above approximation adopted in the index ellipsoid theory, and making a $45^{\circ}$ coordinate rotation back to $x, y$, and $z$, we finally arrive the coupling equations that the light field components satisfy,

$$
\begin{gathered}
\frac{\partial^{2} \tilde{E}_{x}}{\partial^{2} z}-i k_{x} \frac{\partial \tilde{E}_{z}}{\partial z}+k_{x} k_{y} \tilde{E}_{y}+\left[k_{0}^{2}\left(n_{0}-n_{0}^{3} \gamma_{63} E_{0} / 2\right)^{2}-k_{y}^{2}\right] \tilde{E}_{x}=0, \\
\frac{\partial^{2} \tilde{E}_{y}}{\partial^{2} z}-i k_{y} \frac{\partial \tilde{E}_{z}}{\partial z}+k_{x} k_{y} \tilde{E}_{x}+\left[k_{0}^{2}\left(n_{0}+n_{o}^{3} \gamma_{63} E_{0} / 2\right)^{2}-k_{x}^{2}\right] \tilde{E}_{y}=0, \\
\tilde{E}_{z}=\frac{i}{k_{0}^{2} n_{e}^{2}-k_{\perp}^{2}}\left(k_{x} \frac{\partial \tilde{E}_{x}}{\partial z}+k_{y} \frac{\partial \tilde{E}_{y}}{\partial z}\right) .
\end{gathered}
$$

The above equations form a complete description of light propagation in the momentum space. Note that the coefficients of $\tilde{E}_{y}$ in Eq. (4) and $\tilde{E}_{x}$ in Eq. (5) are both electrically tunable with $E_{0}$, which play a key role in our flexible manipulation of polarization singularities. In our simulation, we consider the incident light is a lefthanded circularly polarized Gaussian one, namely, $\mathbf{E}\left(\mathbf{r}_{\perp}, z=0\right)=\exp \left(-r_{\perp}^{2} / 2 s^{2}\right) \hat{\mathbf{e}}_{+}$. With the boundary condition of $\tilde{\mathbf{E}}_{\perp}(0)=\frac{s^{2}}{2 \pi} \exp \left(-k_{\perp}^{2} s^{2} / 2\right) \hat{\mathbf{e}}_{+}$, the second-order partial differential equations (4)-(6) can be solved to obtain the solution for $\tilde{\mathbf{E}}\left(\mathbf{k}_{\perp}, z\right)$.
By performing a Fourier integral toward $\widetilde{\mathbf{E}}\left(\mathbf{k}_{\perp}, z\right)$, we can finally write the optical field in real space as,

$\mathbf{E}\left(\mathbf{r}_{\perp}, z\right)=\int d^{2} \mathbf{k}_{\perp}\left\{\left[\begin{array}{l}c_{1} \\ c_{3} \\ c_{5}\end{array}\right] \exp \left(\sqrt{\lambda_{1}} z+i \mathbf{k}_{\perp} \cdot \mathbf{r}_{\perp}\right)+\left[\begin{array}{l}c_{2} \\ c_{4} \\ c_{6}\end{array}\right] \exp \left(\sqrt{\lambda_{2}} z+i \mathbf{k}_{\perp} \cdot \mathbf{r}_{\perp}\right)\right\},(7)$

in which the coefficients $\lambda_{1}, \lambda_{2}$, and $c_{1} \sim c_{6}$ are given by Eqs. (S14) to (S20) in the supplementary materials. This equation is the starting point of all our calculations. However, normally, the double Fourier integral in Eq. (7) is difficult to be evaluated analytically due to the complex forms of $c_{1} \sim c_{6}$. It can be worked out only for several special inputting fields and in the paraxial limit ${ }^{35}$. We therefore turn to numerical method to calculate it in this paper.

In respect of numerical solutions, the main difficulty is that the integrals in Eq. (7) are highly oscillating, where the traditional quadrature methods are invalid ${ }^{36,41}$. To overcome this difficulty, we have introduced in Ref. [36] an accurate and numerically cheap method based on asymptotic expansion theory. Specifically, one need to calculate a typical oscillatory integral in Eq. (7), $I(z)=\iint g\left(k_{x}, k_{y}\right) \exp \left[i z f\left(k_{x}, k_{y}\right)\right] d k_{x} d k_{y}$, where the function $g$ can be $c_{1} \sim c_{6}$, the function $f$ can be $f_{1} \sim f_{2}$ with $f_{1,2}=\sqrt{-\lambda_{1,2}}+\frac{x}{z} k_{x}+\frac{y}{z} k_{y}$. This double integral can be well approximated by asymptotic expansion ${ }^{37,41}$ (or equally the stationary phase method, see the supplementary materials), when the value of $z$ is sufficiently large. The essence of the asymptotic expansion theory is that the dominated contribution to the highly oscillatory integral $I(z)$ only comes from the small regions in the vicinity of certain critical points ${ }^{37,41}$. The most important critical points are the stationary points of function $f\left(k_{x}, k_{y}\right)$ inside the domain of integration, which satisfy the condition $\frac{\partial f}{\partial k_{x}}=\frac{\partial f}{\partial k_{y}}=0$. The other kinds of critical points are defined on the boundary curve of integration and thus are unimportant here, for the boundary of Fourier integral of Eq. (7) is actually at infinity.

Based on this observation, our idea of approximation to Eq. (7) is as follows: Instead of integrating over the whole Fourier $k$ space, we only numerically integrate over small (and important) regions near the stationary points of functions $f_{1,2}$ and throw away the contributions from the other oscillatory regions ${ }^{36}$. The part of the integral that is thrown away decays exponentially fast as the oscillation increases ${ }^{41}$. A consequent question is how to choose the domain of integration. We have verified that a square integrating boundary yields the smallest error with respect to the analytical solution ${ }^{36}$. We illustrate a schematic domain of integration in Fig. 5, where the domain is denoted by the gray area centered at the stationary point $S$. The accuracy of this method can be further improved by a coarse-grained average treatment. For example, we can choose ten equally spaced values $d_{k}$ in one oscillating period of integrand, e.g., in the slant-line shading regions between two red vertical lines in Fig. 5(a) or two red squares in Fig. 5(b), calculate the integral using each $d_{k}$ as the width of integrating domain, and treat their average as the final result. In this way, the highly oscillating part far away from the critical point is canceled, leaving the most important contribution from the small area close to the critical point.

Our calculating procedure is as follows: We first numerically find the stationary points of functions $f_{1,2}$, determinate the domains of integration by counting the periods of $\exp \left(i z f_{1,2}\right)$, perform the numerical integration in small regions using GaussKronrod quadrature, and finally do average over one oscillating period to get the final result. More detailed discussions about the validity and accuracy of this numerical method can be found in the supplementary materials. After computing the optical fields involving the Pockels effect, we can then explore the dynamics of polarization 
singularities. It is noted that our method can be extended to any crystals of arbitrary point group with the biaxial anisotropy is induced by the Pockels effect, Kerr effect or other nonlinear optical effects.

1. Azzam, R. M. A. \& Bashara, N. M. Ellipsometry and Polarized Light (NorthHolland, Amsterdam, 1997).

2. Nye, J. F. Polarization effects in the diffraction of electromagnetic waves: the role of disclinations. Proc. R. Soc. Lond. A 387, 105-132 (1983).

3. Nye, J. F. Lines of circular polarization in electromagnetic wave fields. Proc. R. Soc. Lond. A 389, 279-290 (1983).

4. Nye, J. F. Natural Focusing and Fine Structure of Light (Institute of Physics, Bristol, 1999).

5. Dennis, M. R., O’ Holleran, K. \& Padgett, M. J. Singular optics: optical vortices and polarization singularities. Prog. Opt. 53, 293-363 (2009).

6. Molina-Terriza, G., Torres, J. \& Torner, L. Twisted photons. Nature Phys. 3 , 305-310 (2007).

7. Yao, A. M. \& Padgett, M. J. Orbital angular momentum: origins, behavior and applications. Adv. Opt. Photon. 3, 161-204 (2011).

8. Leach, J., Dennis, M. R., Courtial, J. \& Padgett, M. J. Laser beams: Knotted threads of darkness. Nature 432, 165 (2004).

9. Leach, J., Dennis, M. R., Courtial, J. \& Padgett, M. J. Vortex knots in light. New J. Phys. 7, 55 (2005).

10. Dennis, M. R., King, R. P., Jack, B., O’Holleran, K. \& Padgett, M. J. Isolated optical vortex knots. Nature Phys. 6, 118-121 (2010).

11. Dennis, M. R. Opt. Commun. Polarization singularities in paraxial vector fields: morphology and statistics. 213, 201-221 (2002).

12. Flossmann, F., O’Holleran, K., Dennis, M. R. \& Padgett, M. J. Polarization Singularities in 2D and 3D Speckle Fields. Phys. Rev. Lett. 100, 203902 (2008).

13. Berry, M. V., Dennis, M. R. \& Lee, R. L. Polarization singularities in the clear sky. New J. Phys. 6, 162 (2004).

14. Chen, Y. F., Lu, T. H. \& Huang, K. F. Hyperboloid Structures Formed by Polarization Singularities in Coherent Vector Fields with LongitudinalTransverse Coupling. Phys. Rev. Lett. 97, 233903 (2006).

15. Lindfors, K. et al. Local polarization of tightly focused unpolarized light. Nature Photon. 1, 228-231 (2007).

16. Cardano, F., Karimi, E., Marrucci, L., de Lisio, C. \& Santamato, E. Generation and dynamics of optical beams with polarization singularities. Opt. Express 21, 8815-8820 (2013).

17. Fission (biology). http://en.wikipedia.org/wiki/Binary_fission (1st October 2013)

18. Egorov, Y. A., Fadeyeva, T. A. \& Volyar, A. V. The fine structure of singular beams in crystals: colours and polarization. J. Opt. A: Pure Appl. Opt. 6, S217 (2004).

19. Flossmann, F., Schwarz, U. T., Maier, M. \& Dennis, M. R. Polarization Singularities from Unfolding an Optical Vortex through a Birefringent Crystal. Phys. Rev. Lett. 95, 253901 (2005).

20. Desyatnikov, A. et al. Spatially engineered polarization states and optical vortices in uniaxial crystals. Opt. Express 18, 10848-10863 (2010).

21. Fadeyeva, T. A., Alexeyev, C. N., Anischenko, P. M. \& Volyar, A. V. Engineering of the space-variant linear polarization of vortex-beams in biaxially induced crystals. Appl. Opt. 51, C224-C230 (2012).

22. Skab, I., Vasylkiv, Y., Smaga, I. \& Vlokh, R. Spin-to-orbital momentum conversion via electro-optic Pockels effect in crystals. Phys. Rev. A 84, 043815 (2011).

23. Skab, I. et al. Optical anisotropy induced by torsion stresses in $\mathrm{LiNbO}_{3}$ crystals: appearance of an optical vortex. J. Opt. Soc. Am. A 28, 633-640 (2011).

24. Chen, L., Zheng, G., Xu, J., Zhang, B. \& She, W. Electrically controlled transfer of spin angular momentum of light in an optically active medium. Opt. Lett. 31, 3474-3476 (2006).

25. Chen, L., Zheng, G. \& She, W. Electrically and magnetically controlled optical spanner based on the transfer of spin angular momentum of light in an optically active medium. Phys. Rev. A (Rapid communications) 75, R061403 (2007).

26. Chen, L. \& She, W. Electro-optically forbidden or enhanced spin-to-orbital angular momentum conversion in a focused light beam. Opt. Lett. 33, 696-698 (2008).
27. Chen, L. \& She, W. Electrically tunable and spin-dependent integer or non-integer orbital angular momentum generator. Opt. Lett. 34, 178-180 (2009).

28. Barnett, S. M. Optical angular-momentum flux. J. Opt. B: Quantum Semiclass. Opt. 4, S7-S16 (2002).

29. Ciattoni, A., Cincotti, G. \& Palma, C. Angular momentum dynamics of a paraxial beam in a uniaxial crystal. Phys. Rev. E 67, 036618 (2003).

30. Brasselet, E. et al. Dynamics of optical spin-orbit coupling in uniaxial crystals. Opt. Lett. 34, 1021 (2009).

31. Lu, X. \& Chen, L. Spin-orbit interactions of a Gaussian light propagating in biaxial crystals. Optics Express 20, 11753-11766 (2012).

32. Loffler, W., Aiello, A. \& Woerdman, J. P. Observation of Orbital Angular Momentum Sidebands due to Optical Reflection. Phys. Rev. Lett. 109, 113602 (2012).

33. Yariv, A. \& Yeh, P. Optical Waves in Crystals (Wiley, New York, 1984).

34. Shen, Y. R. The Principles of Nonlinear Optics (Wiley-Interscience, New York, 1984).

35. Ciattoni, A., Crosignani, B. \& Di Porto, P. Vectorial theory of propagation in uniaxially anisotropic media. J. Opt. Soc. Am. A 18, 1656-1661 (2001).

36. Lu, X. \& Chen, L. Anisotropic dynamics of optical vortex-beam propagating in biaxial crystals: a numerical method based on asymptotic expansion. Opt. Express 21, 8493-8507 (2013)

37. Born, M. \& Wolf, E. Principles of Optics (Pergamon, Oxford, 1959).

38. Wooten, L. et al. A review of lithium niobate modulators for fiber-optic communications systems. IEEEJ. Sel. Top. Quantum Electron. 6, 69-82 (2000).

39. Angelsky, O., Mokhun, A., Mokhun, I. \& Soskin, M. The relationship between topological characteristics of component vortices and polarization singularities. Opt. Commun. 207, 57-65 (2002).

40. Freund, I. Poincaré vortices. Opt. Lett. 26, 1996-1998 (2001)

41. Wong, R. Asymptotic Approximations of Integrals (SIAM, Philadelphia, 2001).

\section{Acknowledgments}

This work is supported by the National Natural Science Foundation of China (NSFC) (Nos. 11104233 and 11004164), the Program for New Century Excellent Talents in University of China (NCET-2013-0495), the Doctoral Fund of Ministry of Education of China (No. 2011012112003), and the Fundamental Research Funds for the Central Universities (Nos 2011121043 and 2012121015).

\section{Author contributions}

L.C. conceived the theory, X.L. and L.C. performed the numerical calculations. X.L., L.C., Z.W. and W.Z. analyzed the numeric data. L.C. supervised the project. All authors discussed the results and contributed to the writing of the manuscript.

\section{Additional information}

Supplementary information accompanies this paper at http://www.nature.com/ scientificreports

Competing financial interests: The authors declare no competing financial interests.

How to cite this article: Lu, X.C., Wu, Z.W., Zhang, W.H. \& Chen, L.X. Polarization singularities and orbital angular momentum sidebands from rotational symmetry broken by the Pockels effect. Sci. Rep. 4, 4865; DOI:10.1038/srep04865 (2014).

This work is licensed under a Creative Commons Attribution-NonCommercialNoDerivs 3.0 Unported License. The images in this article are included in the article's Creative Commons license, unless indicated otherwise in the image credit; if the image is not included under the Creative Commons license, users will need to obtain permission from the license holder in order to reproduce the image. To view a copy of this license, visit http://creativecommons.org/licenses/by-nc-nd/3.0/ 